\title{
An All-Optical Grooming Switch to Interconnect Access and Metro Ring Networks
}

\author{
J. Leuthold, W. Freude, S. Sygletos, P. Vorreau, R. Bonk, D. Hillerkuss \\ Institute of High-Frequency and Quantum Electronics, University of Karlsruhe, 76131 Karlsruhe, Germany \\ Tel: +49 721 808-2481, Fax: +49 721 608-9098, \\ Project web page at http://www.ihq.uni-karlsruhe.de/research/projects/TRIUMPH/ \\ I. Tomkos, A. Tzanakaki, C. Kouloumentas \\ Athens Information Technology, Athens, Greece \\ D. J. Richardson, P. Petropoulos, F. Parmigiani \\ Optoelectronics Research Centre, University of Southampton, United Kingdom \\ A. Ellis, D. Cotter, S. Ibrahim, R. Weerasuriya \\ Photonic Systems Group, Department of Physics, University College Cork, Cork, Ireland
}

\begin{abstract}
A novel switch concept is introduced that offers transparent optical grooming of 10 and $40 \mathrm{~Gb} / \mathrm{s}$ traffic in an access network onto a metro core ring network operated at $130 \mathrm{~Gb} / \mathrm{s}$ traffic. Key functionalities of the router are the traffic aggregation with time-slot interchanging (TSI) functionality, the TDM to WDM demultiplexing of the high-speed channel into lower bit-rate tributaries as well as multi-wavelength all-optical 2R regeneration of several highest-speed signals.
\end{abstract}

\section{INTRODUCTION}

Optical transparency is widely recognized to play a central role in next generation optical networks and offer significant networking advancements in terms of performance and cost [1]. The elimination of the expensive OE-O equipment and the enhanced scalability of the optical technology are expected to significantly reduce the capital costs, whilst operational cost savings are also expected due to the faster provisioning times, and the reduced power requirements. Although first transparent network deployments are basically transparent point-topoint links with OADMs at intermediate nodes the trend is towards so-called islands of transparency and potentially towards fully meshed transparent networks with reconfigurable OXCs [2]. Such transparent solutions have recently been developed into commercially available products [3]. A notable example is the Verizon Business network, which consists of several regional systems that are connected via ROADMs/OXCs and extend over the entire geographical area of the continental United States [4].

Although most of the activities have been focused on developing the core part of transparent networks [5]-[7], little effort has been made at the edges. At edge nodes any proposed solution should be competitive in terms of both performance and cost to legacy SONET/SDH expansions. This means that edge nodes should be able not only to perform the traditional switching/routing and protection functionalities, but also offer transparent mechanisms for traffic aggregation and grooming, i.e. sub-wavelength switching granularity. This will assist in simplifying the network architecture and eliminate unnecessary opto-electronic conversions having a direct impact on the cost of the overall network system.

The main goal of the European project TRIUMPH is the implementation of the first edge switch with grooming functionality to enable full transparency between circuit or burst switched lower bit-rate access networks and higher-bit rate core ring network. The network scenario for which the TRIUMPH router will be developed is depicted in Fig. 1. It shows two access networks carrying 10 to $40 \mathrm{~Gb} / \mathrm{s}$ data bursts that are interconnected with each other but may also be aggregated to higher-speed $(130 \mathrm{~Gb} / \mathrm{s})$ data-bursts on the Regional Metro Ring. Viceversa, data bursts from the Regional Metro Ring might be dropped to one of the access rings.

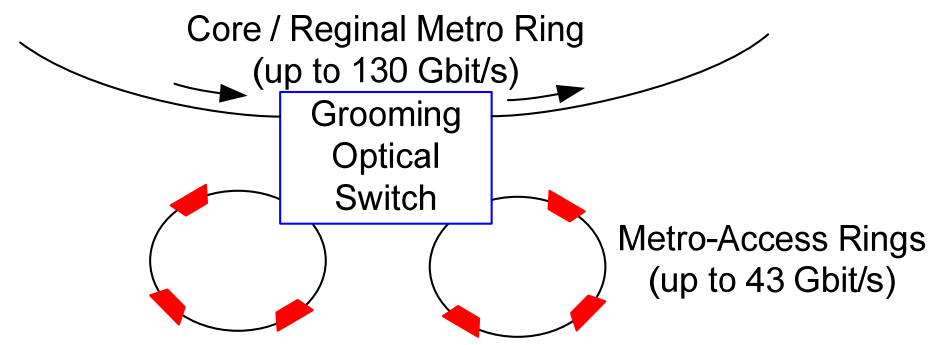

Fig. 1. Network scenario: several Metro-Access rings are interconnected to a Core/Regional Metro ring network. Thereby lower speed traffic has to be groomed to higher speed traffic and vice-versa. 


\section{IMPLEMENTATION OF THE GROOMING SWITCH}

The architecture of the TRIUMPH grooming switch to enable transparency at edge nodes is depicted in Fig. 2. The switch interconnects two access rings - each carrying three $43 \mathrm{~Gb} / \mathrm{s}$ WDM channels with a metro core ring with two $130 \mathrm{~Gb} / \mathrm{s}$ channels. The switch itself comprises of a MEMS switch fabric, a WDM-to-OTDM unit, a OTDM-to-WDM unit and a signal regenerator.

The switch works as follows: Traffic from any of the access rings is switched by means of an optical crossconnect switch (a MEMS switch) onto either access ring or via the Add path onto the core ring. A WDM-toOTDM unit in the add path of the optical grooming switch retimes the incoming asynchronous data bursts to a local clock and all-optically converts the signal to the short high-speed pulses of the OTDM channel at a desired wavelength. This WDM-to-OTDM unit is subsequently called Asynchronous Digital Optical Regenerator (ADORE) [8][9]. This WDM-to-OTDM conversion is performed for each tributary. The time-slot assignment is thereby performed by the selection of the output channel within the MEMS switch. This guarantees full time-slot interchanging functionality. Conversely, an OTDM channel may be dropped and any of the three OTDM tributaries may be mapped onto either of the access rings or back onto the core ring. The OTDM to WDM conversion thereby is performed all-optically by a single device. To guarantee the quality of the traffic in the core ring alloptical multi-wavelength capable signal regenerators operating at $130 \mathrm{~Gb} / \mathrm{s}$ traffic are added in the core ring.

Core/Regional Metro Ring

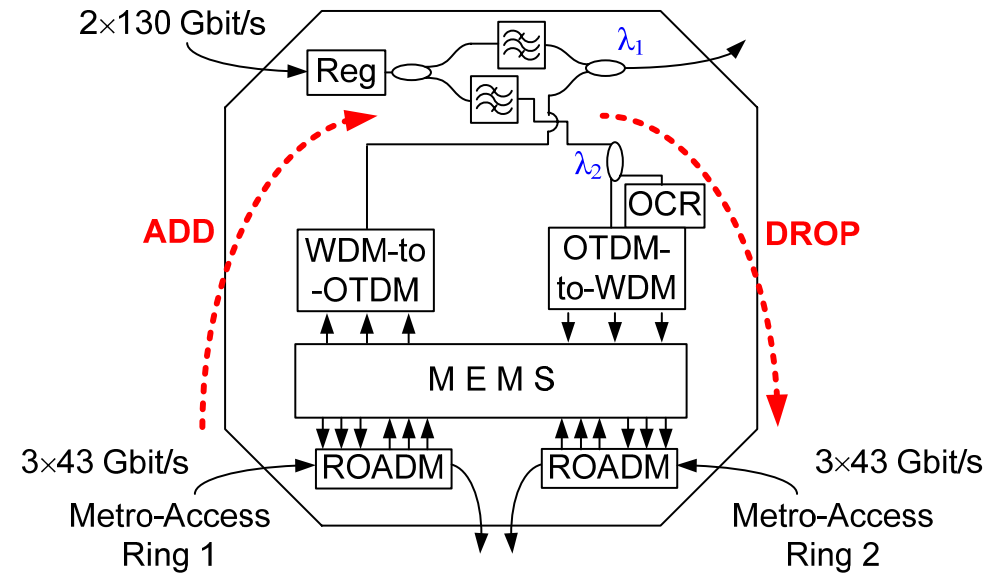

Fig. 2. Grooming optical switch node configuration: several Metro-Access Rings are interconnected to a Core/Regional Metro Ring network. Thereby lower speed traffic has to be groomed to higher speed network rings and vice-versa.

\section{THE TRIUMPH SWITCH BUILDING BLOCKS}

\subsection{The WDM-to-OTDM unit (ADORE)}

OTDM multiplexing of several lower bit-rate channels requires shortening the pulse-widths of the tributaries, mapping of all tributaries onto a common wavelength and introducing precise relative timing delays between the signals so that pulses from neighbouring channels do not overlap after optical multiplexing. The latter is of particular importance, since incoming low speed data signals from multiple sources usually have different clock frequencies and environmentally induced changes in propagation delays.

The functional diagram of the ADORE [9] scheme is shown in Fig. 3. In the ADORE, an incoming data burst is simultaneously sampled at four different clock phases - each delayed by T/4 bit-slot durations with respect to its neighbour. The clocks are then led through four optical gates that are controlled by a $43 \mathrm{~Gb} / \mathrm{s}$ tributary $\left(\mathrm{f}_{\mathrm{R}}\right)$. One of the four gated clock pulses will typically pass the gate synchronously with the incoming signal. Consequently, the power from this clock pulse is dominant. Behind the gates the relative time delays are reset. The four gating clocks (one of which is dominant) are now all in sync with the local clock. They carry the wavelength of the local clock and are shortened to the duration of the clock pulse. While one now could pick the one channel with the highest energy for subsequent processing, we have constructively combined two and two of the four output channels into two outputs. The optimum channel is detected by mixing the local clock with the incoming data signal electronically. The mixer output voltage is proportional to the cosine of the phase difference between the input data signal and the local clock. This voltage is fed into a control circuit for selecting either combined channel A or B, depending on which channel is regenerated best. The schematic of Fig. 3 might lead to the wrong perception, that this scheme demands for a lot of devices. Yet, in our real implementation simplification has been achieved by interferometrically combining neighbouring clock phases which are encoded with the same bit of the input signal. This way only one gate is needed [9]. 
Lastly, one should mention that for combining three $43 \mathrm{~Gb} / \mathrm{s}$ tributaries into one $130 \mathrm{~Gb} / \mathrm{s}$ OTDM signal one requires three ADORE units. The outputs of which need to be interleaved in appropriate time delays.

(a)

(b)
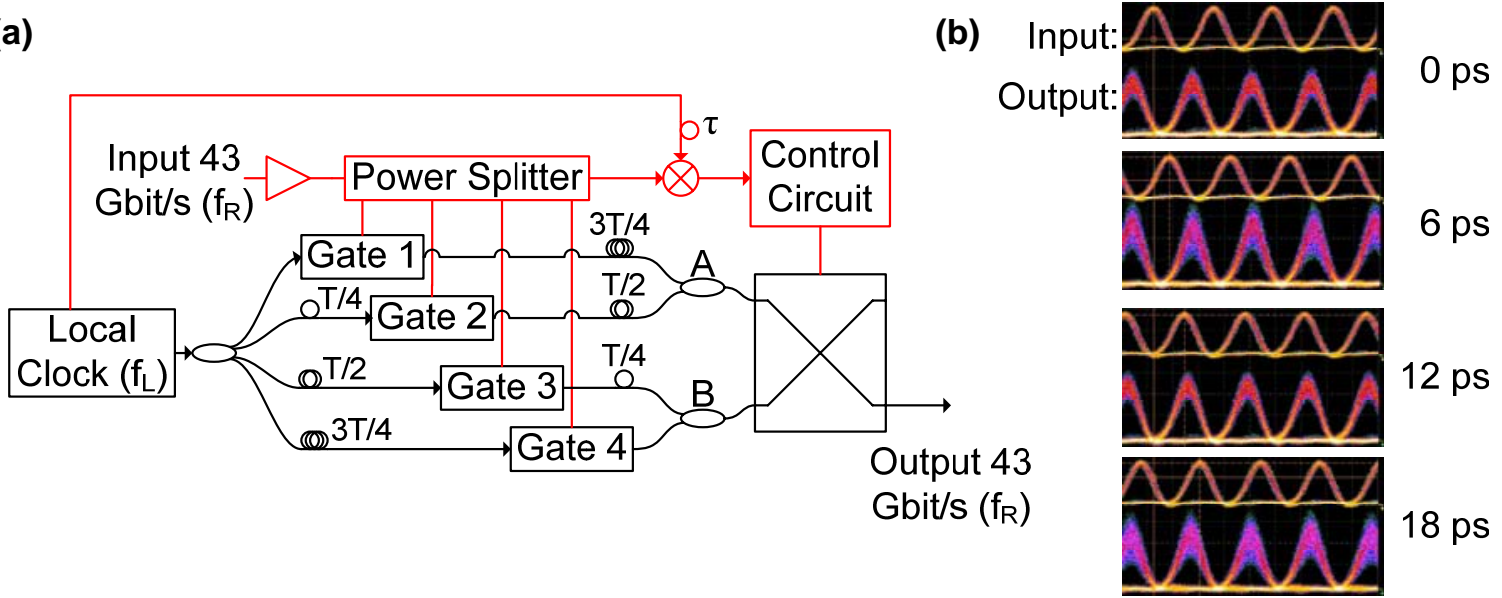

Fig. 3. (a)Functional diagram of the ADORE. The device is synchronizing an input $R Z$ signal with a local clock. It maps the information of the input RZ signal onto four copies of the clock-each delayed by T/4. The one clock that is best in sync with the input signal is then becoming the new output signal. (b) Measured eye diagrams of the input and output signal for four different delays, showing that resynchronization works for any relative delay of input with respect to the local clock.

\subsection{The OTDM-to-WDM unit}

The scheme of the $130 \mathrm{~Gb} / \mathrm{s}$ TDM to three $43 \mathrm{~Gb} / \mathrm{s}$ WDM demultiplexer is shown in Fig. 4. Three $43 \mathrm{GHz}$ clocks $\left(\lambda_{1,2,3}\right)$ are temporally aligned to different time slots of the TDM signal and are launched into the input port of a nonlinear optical loop (NOLM). If a TDM pulse (at wavelength $\lambda_{0}$ ) is present ('ones'), a nonlinear phase shift is induced onto the copropagating clock pulse that is temporarily aligned with the respective pulse. The induced phase shift will lead to a constructive interference of the co- and counterpropagating clock pulse into the output. This allows to effectively transfer coded information from specific time slots at one wavelength to different wavelengths. For effective multi-wavelength operation of the NOLM an accurate control of the dispersion and dispersion slope of the fiber is needed to ensure a low walk-off and uniform switching efficiency across the whole bandwidth of the clock signals.

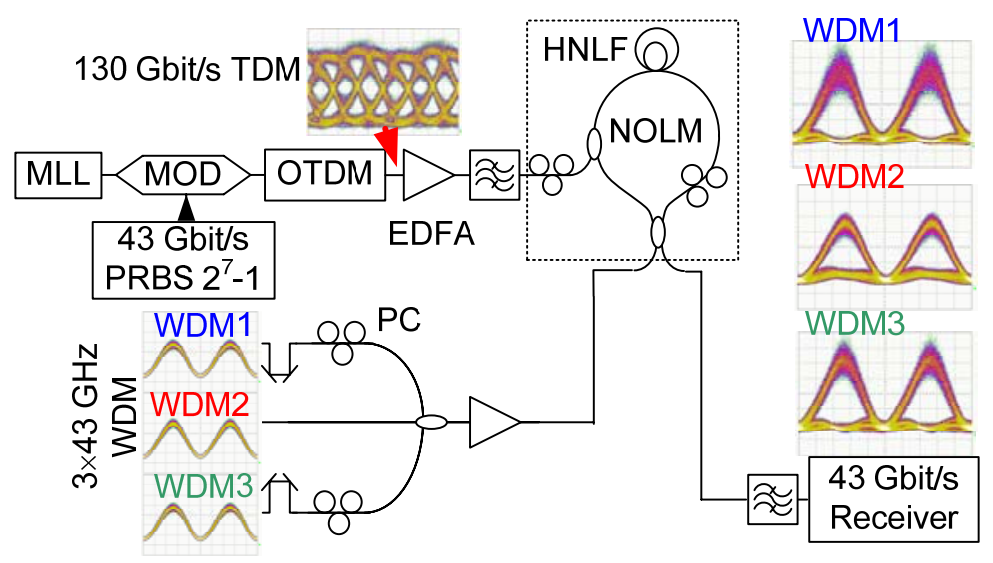

Fig. 4. Experimental setup of the TDM-to-WDM conversion. Insets: Eye diagrams at the input of the system and of the three demultiplexed WDM signals at the system output.

The experiment depicted in Fig. 4 shows how a $128.1 \mathrm{Gbit} / \mathrm{s}$ TDM channel operated at $\lambda_{0}=1539.4$ and generated with a Mode-locked laser (MLL) is mapped onto three clocks signals at wavelengths $\lambda_{1}=1547.8 \mathrm{~nm}$, $\lambda_{2}=1552.5 \mathrm{~nm}$ and $\lambda_{3}=1557.4 \mathrm{~nm}$. The power of the clock signals in the loop were $\sim 20 \mathrm{dBm}$ and the TDM signal was $\sim 23 \mathrm{dBm}$, before entering the NOLM via a 3dB coupler. The HNLF fabricated by Furukawa Electric Co., Japan, had a length of $310 \mathrm{~m}$, dispersion of $-0.31 \mathrm{ps} / \mathrm{nm} / \mathrm{km}$, dispersion slope of $0.0031 \mathrm{ps} / \mathrm{nm}^{2} / \mathrm{km}$, nonlinear coefficient of $20 / \mathrm{W} / \mathrm{km}$ and loss of $1.21 \mathrm{~dB} / \mathrm{km}$. These fibre parameters gave a maximum walk-off time between the TDM and the WDM signals of 1.8ps. The WDM signals switched by the NOLM were then separated 
by a WDM demultiplexer filter, and individually assessed using a single preamplified receiver, which was constituted of a variable attenuator, an erbium doped fiber amplifier (EDFA), a 1nm filter and a 40GHz receiver.

Fig. 4(b) shows the spectral traces at the output port of the NOLM, and eye diagrams show the three simultaneously switched WDM channels. A high extinction ratio of the switched eye was guaranteed by a controlled amount of optical bias (phase difference) between signals propagating through the loop in opposite directions, which was generated by the birefringence of the NOLM, and tuned using the internal polarization controllers.

\subsection{The Multi-Wavelength Regenerator}

To demonstrate multi-wavelength regeneration with two and three $43 \mathrm{~Gb} / \mathrm{s}$ channels, respectively, we built the setup shown in Fig. 5. It comprises a three channel transmitter, a signal decorrelation stage, the regenerator and a preamplified receiver.

The transmitter consists of three DFB lasers on a $600 \mathrm{GHz}$ grid, amplitude modulated at $43 \mathrm{~Gb} / \mathrm{s}$ by a $2^{31}-1$ long PRBS. A series of optical delay lines (ODL) and variable optical attenuators (VOA) between two arrayedwaveguide gratings (AWG) provided some pattern decorrelation and adjustment of the relative time delays. The subsequent regenerator consists of a high-power optical amplifier, the fiber assembly and a $0.53 \mathrm{~nm}$ tuneable optical band-pass filter (OBPF) that allowed for the individual selection of channels. The filter was detuned by $0.6 \mathrm{~nm}$ from the center wavelength. The $5.97 \mathrm{~km}$-long fiber assembly contained five DCF-SMF pairs. The length of each DCF exhibited a maximum differential group delay of 150 ps between the three channels.

In Fig. 5 (b) the bit-error-rate (BER) curves are presented for one of the three channels related to the input, the regenerator output in single-channel operation and the output in dual-channel operation (presence of interfering channel), respectively.
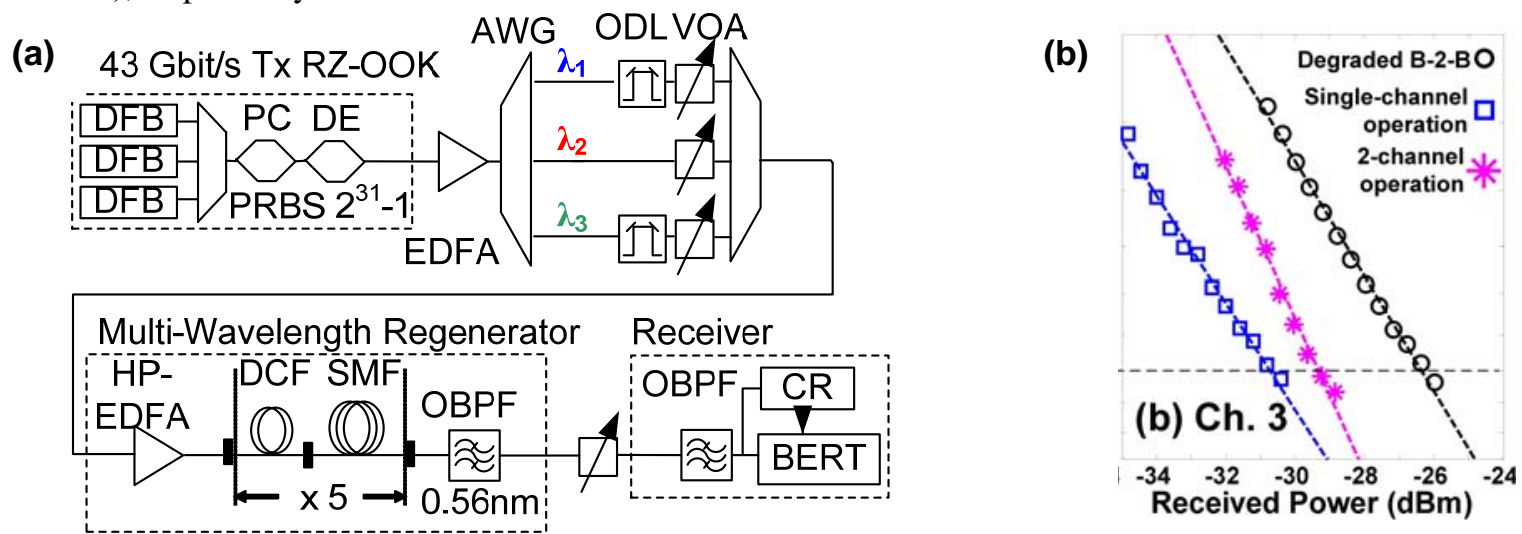

Fig. 5. (a) Experimental setup and configuration of the $2 R$ multi-wavelength regenerator. The transmitter (Tx) generates three $43 \mathrm{~Gb} / \mathrm{s} R Z$ on-off keying signals. The signals are decorrelated by optical delays before guided into the $2 R$ regenerator. The $2 R$ is built as a Mamyschev self-phase modulation regenerator. It comprises five sequences of DCF and SMF segments that are arranged such that cross-phase modulation from one channel onto another is minimized. (b) The bit-error rate (BER) measurements show that the scheme can successfully improve the BER of 2 degraded inpzut signals.

\section{CONCLUSIONS}

In this work we have shown the operation principle and given experimental evidence of the feasability of the first all-optical switch with grooming functionality.

\section{ACKNOWLEDGEMENTS}

This work was supported by the European project TRIUMPH (grant IST-027638 STP) and by the Center for Functional Nanostructures (CFN) of the Deutsche Forschungsgemeinschaft (DFG) within Project A4.4.

\section{REFERENCES}

[1] Siemens Press Release, Oct. 11, 2004;

[2] A.A.M. Saleh; LEOS Summer Topicals on All-Optical Networks, July, 2002, pp. 15.

[3] P. Hofmann, E. E. Basch, S. Gringeri, R. Egorov, D. A. Fishman, W. A. Thompson, Proc. OFC’05, vol. 2, pp. 3, 2005

[4] D. Fishman, D. L. Correa, E. H. Goode, T. L. Downs, A. Y. Ho, A. Hale, P. Hofmann, B. Basch, S. Gringeri, Bell Labs Technical J., vol. 11, no. 2, pp. 55-63, 2006

[5] S. J. B. Yoo, ; IEEE/OSA Journal of Lightwave Technology, Vol.24, No.12, pp. 4468-4492, Dec. 2006 (invited).

[6] D. J. Blumenthal, B. E. Olsson, G. Rossi, T. E. Dimmick, L. Rau, M. Masanovic, O. Lavrova, R. Doshi, O. Jerphagnon, J. E. Bowers, V. Kaman, L. A. Coldren, and J. Barton; J. Lightw. Technol., vol. 18, no. 12, pp. 2058-2075, Dec. 2000.

[7] A. Al Amin et al, ECOC(2006), PDP.Th.4.1.6.

[8] C. W. Chow, A. D. Ellis, and D. Cotter; Opt. Express 15, 8507-8512 (2007).

[9] D. Hillerkuss, A. Ellis, G.Zarris, D. Simeonidou, J. Leuthold, D.Cotter; submitted for publication 